\title{
Effectiveness of Intubating Laryngeal Mask Airway in managing out-of-hospital cardiac arrest by non-physicians
}

\section{Elena-Laura Lemaitre ${ }^{\mathrm{a}, \mathrm{b}^{*}}$ MD, Laurent Tritsch $^{\mathrm{c}}$ MD, Eric Noll ${ }^{\mathrm{b}}$ MD-PhD, Pierre Diemunsch ${ }^{\mathrm{b}}$ MD-PhD, Nicolas Meyer ${ }^{\mathrm{d}, \mathrm{e}, \mathrm{f}}$ MD-PhD}

a. Emergency Department, Hôpitaux Universitaires de Strasbourg, University of Strasbourg, Strasbourg, France

b. Department of Anaesthesiology, Critical Care and Prehospital Emergency Medicine, Hôpitaux Universitaires de Strasbourg, University of Strasbourg, Strasbourg, France

c. Fire and Rescue Department Bas-Rhin, Strasbourg, France

d. Laboratory of Biostatistics, Faculty of Medicine, Strasbourg, France

e. ICUBE UMR 7357, University of Strasbourg, Strasbourg, France

f. Public Health Department, Département de Santé Publique, GMRC, Hôpitaux Universitaires de Strasbourg, Strasbourg, France

* Corresponding author at: Service d'Accueil des Urgences Adultes, 1 avenue Molière, 67200 Strasbourg, France E-mail address: elenalaura.lemaitre@gmail.com (Elena-Laura Lemaitre)

\section{Abstract}

Aim of the study: The role of supraglottic devices in airway management in out-of-hospital cardiac arrest (OHCA) remains controversial. The aim of this study was to evaluate the feasibility and 
effectiveness of intubation through the Intubating Laryngeal Mask Airway (ILMA) when used by prehospital emergency nurses in the setting of OHCA.

Methods: We conducted a prospective, observational trial during 12 years by the Fire Department and prehospital emergency service of the health district of Strasbourg, France. The primary outcome was the success rate of ventilation after intubation through the ILMA, while the secondary outcomes were the success rate of ventilation after insertion of the ILMA and complications related to ILMA placement and intubation. Factors associated with successful intubation were also studied.

Results: During the study period, 1464 ILMA placements were attempted by emergency nurses during OHCA. Ventilation was possible in 1250 patients (85.38\%) after ILMA placement and in 1078 patients (73.63\%) after intubation. Regurgitation of gastric contents occurred in 237 (16.18\%) patients, mostly during basic life support. Two factors were predictive of a successful tracheal intubation: the performance of the Chandy maneuver OR=2.91 (CI:2.07-3.97) and the number of attempts at intubation $\mathrm{OR}=1.95$ (CI:1.43-2.61). Conversely, the number of attempts at ILMA insertion was predictive of an intubation failure $\mathrm{OR}=0.11$ (CI:0.07-0.17).

Conclusion: The success rate of intubation through the ILMA was high. After ILMA placement, ventilation was possible in 1250 patients $(85.38 \%)$ and in 1078 patients $(73.63 \%)$ after intubation.

\section{Keywords:}

Out-of-hospital cardiac arrest; emergency medical services; airway management; endotracheal intubation; supraglottic airway devices; paramedics

\section{INTRODUCTION}


In case of an out-of-hospital cardiac arrest (OHCA), the European Resuscitation Council [1] and American Heart Association [2] recommend the endotracheal intubation (ETI) for securing the airways, but it should be attempted only if the healthcare provider is properly trained and has regular, ongoing experience with the technique. In the absence of personal skills in ETI, a supraglottic airway device (SGA) or a bag mask ventilation (BMV) are two acceptable alternatives. The optimal airway management in this setting remains controversial [1]. A multicenter randomized clinical trial comparing BMV versus ETI in 2043 patients with OHCA [3] came to a non-conclusive result.

The ETI is considered the optimal method of managing the airway during OHCA [4]. There is evidence that, without adequate training and experience, the incidence of complications, such as oesophageal intubation (2.4-17\% in studies involving paramedics) [5-9] and dislodgement is unacceptably high. Prolonged attempts at ETI are harmful, the interruption of chest compressions will compromise coronary and cerebral perfusion. In a study of prehospital intubation by paramedics during 100 OHCA the total duration of the interruptions in CPR associated with ETI attempts was $110 \mathrm{~s}$ and in $25 \%$ of cases the interruptions were more than 3 min [10]. Intubation success rates correlate with the intubation experience attained by paramedics [11]. The intubation failure rate is $50 \%$ in prehospital systems with providers who do not perform intubation frequently [12-13]. Tracheal intubation is a difficult skill to acquire and maintain. Anaesthesia residents required about 125 intubations in the operating room setting before they were able to achieve an intubation success rate of $95 \%$ [14].

Although, BMV appears to be easier to perform [15], it may be difficult to ensure and the risk of regurgitation and pulmonary aspiration is higher [16].

There are published studies on the use of SGA devices during CPR, but none of these studies have been powered adequately to enable survival to be studied as a primary endpoint [17]. The SGA devices are easier to use and, unlike ETI, can be inserted without interrupting chest compressions. Moreover, the learning curve for the insertion of the intubating laryngeal mask is reported to be shorter [18]. The Intubating Laryngeal Mask Airway (ILMA, Fastrach ${ }^{\mathrm{TM}}$ ) is a device to be used in management of difficult airway. Investigations of the ILMA in the prehospital setting when used by paramedics are scarce [19-21].

The aim of this study was to evaluate the effectiveness and feasibility of intubation through the ILMA when used by the non physicians during OHCA.

\section{METHODS}


We conducted a prospective, observational trial between 1 January 2006 and 31 December 2017 by the Fire Department (FD) and prehospital Emergency Department (ED) of the health district of Strasbourg, Bas-Rhin, France.

\section{1. Ethics}

This study was approved by the Institutional Research Ethics Board of the University of Strasbourg. The Ethics Committee waived the need for informed consent because the study was not randomized and assessed a device routinely used in the practice of the Emergency Service.

\section{2. Organisation of prehospital emergency care}

Bas-Rhin counted 1,116,658 people in 2015, according to INSEE [22] in $4755 \mathrm{~km}^{2}$ with a density of 236.47 inhabitants per $\mathrm{km}^{2}$, which is twice the French average. The department has 5 hospitals with an ED and 8 mobile emergency medical teams (Service d'Aide Médicale Urgente: SMUR).

In case of OHCA, a primary care ambulance with a team of three emergency medical technicians (EMT) firemen on board, a SMUR consisting of an EMT, a nurse and a physician, are available for prehospital patients. Additionally, a FD nurse is available when the location of the intervention is in rural areas of the district because the response time of SMUR is more than ten minutes.

\section{3. Protocol for airway management}

This study included all consecutive OHCA patients for whom a FD nurse used an ILMA for securing the airways in the frame of the predefined standardized protocol for which they were trained.

1. The primary care fireman team is responsible for conducting basic life support (BLS) according to international guidelines including chest compressions, BMV and defibrillation if necessary.

2. Upon arrival of the FD nurse on the scene, he/she is in charge of airway management and drugs administration. As per protocol, the nurse has to insert ILMA, unless the patient has trismus or a small mouth opening and then try to ventilate through the ILMA. The size of the ILMA is chosen according to the recommendations of the manufacturer. Sedatives and neuromuscular blocking agents are not administrated (patients in cardiac arrest). A proper lubrication of the lumen of the ILMA was emphasized to facilitate the passage of the tracheal tube (TT). The FD nurse can use the Chandy maneuver to facilitate the ventilation and/or TT insertion.

3. If the placement of the ILMA is not successful, the FD nurse has to remove it and to resume the bag-mask ventilation.

4. When ventilation through ILMA is effective, a wire reinforced TT is inserted through the 
ILMA. To confirm the tracheal intubation, clinical criteria were used (observing bilateral chest expansion, auscultation using a stethoscope).

5. If the tracheal intubation through the ILMA is not possible or ventilation through TT proves ineffective, the nurse has to remove the TT and continue to ventilate the patient through the ILMA until the arrival of the medical team.

6. Peripheral intravenous catheter insertion and drugs administration follow airway control.

\section{4. ILMA insertion training course}

Participants were trained to insert the ILMA, which consisted of a 20 minutes video didactic presentation followed by the practice on a mannequin (Resusci-Anne Sim, Laerdal Medical, Stavanger, Norway). Three successful insertions of an ILMA size 5 and performance of blind tracheal intubation with a wire reinforced TT size 7 were required to pass the course.

A refresher session had to be attended by all nurses every year. Proficiency using the ILMA was tested after both the initial training and refresher sessions.

Our choice is based on evidence-based education from literature. Several studies have demonstrated that simulation-based education allows transfer of learnt skills from the simulated environment to the clinical setting, both for procedural skills and non-technical skills.

\section{5. Collecting data}

Data recorded were the date and time of the OHCA, the pattern, the departure and arrival time of the FD nurse, location of the intervention, the characteristics of the patient, presence and timing of regurgitation, ILMA and TT size, the quality of the ventilation through the ILMA and after intubation, number of attempts at ILMA and TT insertions and the difficulties encountered.

\section{6. Outcomes}

II.6.1. The primary outcome was the success rate of intubation through the ILMA when used by the non physicians in OHCA.

II.6.2. The secondary outcomes were the success rate of ventilation after insertion of the ILMA and complications related to ILMA placement and intubation.

Factors associated with successful intubation were also studied.

\section{7. Statistical analysis}


The data were analyzed according to the Bayesian paradigm. Categorical data are described as frequency $(\%)$. Count data are described after categorization but were used as count in the subsequent modeling.

A sample of 1450, approximately the expected sample size, would provide us with a power of $89 \%$ to detect an OR of 1.4 for a binary predictor and a power of more than $93 \%$ to detect an OR of 1.2 for a continuous predictor under the assumption that, in both situations, the success rate in the reference group was $50 \%$. This value $(50 \%)$ was chosen because it maximizes the variance and gives thus a conservative estimation of the power.

The primary and secondary outcomes were each modeled with a multiple logistic regression. The selection of the final model was based on a stepwise descending selection based on DIC combined with the clinical relevance of the variable to include or to exclude. For this outcomes analyses, the priors were defined before the study and were based on a generic prior stating that the OR is a priori in the interval [1/150-150]. A random effect was included to take account of the nurse cluster effect, each nurse in this study being implied for one or several cases. The OR are provided with their point estimate and $95 \%$ posterior credibility interval and the probability that the OR is larger than 1 or in a respecified range of values. A large (near 1) or a small (near 0) probability, both suggest an effect.

We remind that the Bayesian paradigm does not use the (frequentist) p-value and that the probability of exceeding that the probability, for a parameter of interest, of exceeding to given threshold must not be confused with a p-value.

Missing data were replaced using multiple imputation. For count data, the missing data were imputed as drawn from a Poisson distribution in which the lambda parameter was equal to the mean of the observed data. For categorical data, a Dirichlet distribution was used for which the observed distribution of the non-missing data was used as parameters.

A burn-in of 5000 iterations, followed by 100000 iteration was used for each analysis. Convergence of the McMC sample chain was checked graphically. Convergence was observed in each case. All computations were done with R 3.2.2 and JAGS statistical softwares with all the required additional packages (R Core Team (2016). R: A Language and environment for statistical computing. R foundation for Statistical Computing. Vienna, Austria, 2014. URL: https://www.Rproject.org/).

\section{RESULTS}




\section{III.1. Patients and operators demographics}

One hundred and sixty two nurses completed the training course and provided cares to 1464 patients during the study period (Fig1). In 210 cases, ventilation was ineffective and in 4 cases we have incomplete data. In the other 1250 cases, the ventilation through the ILMA was effective. From these 1250 cases (we excluded 56 cases: 2 ROSC and 54 dead pronounced) in 1294 cases the nurses tried to introduce the tracheal tube.

The number of male patients was twice as large as the number of females with a sex ratio of 1.86 . The average age of patients was 67.59 years (SD 16.77). More than $50 \%$ of patients were older than 70 (Table 1).

A total of $952(65 \%)$ interventions were performed during the day (between 7a.m. and 7p.m.) and $492(34 \%)$ at night (between 7p.m. and 7a.m.). The median response time from the call to the arrival of the FD nurse on scene was 11.9 minutes (SD 5.86), with a median of 11 minutes.

On average, the FD nurses performed 9 interventions, with a minimum of 1 and a maximum of 141. The women FD nurses performed a total of 801 interventions ( 8 interventions on average) and the men performed 657 interventions (10 interventions on average). Women FD nurses outnumbered men, with a sex ratio male/female $=0.64$. Half of the FD nurses (women and men) completed more than 4 interventions (Table 2).

On average, a FD nurse received 5.39 training sessions (SD 3.25). Half of the interventions concerned FD nurses who had received 5 training sessions. In 35 cases (2.39\%) intubation was considered difficult.

\section{2. Primary outcome}

The intubation success rate was $73.63 \%$ (1078 of 1464 cases). We counted 321 failures of intubation (210 failures of ILMA insertion and 111 failures of ventilation through the TT). After intubation, the ventilation was effective in 1020 (69.67\%) cases after the first attempt whereas only in $50(3.41 \%)$ cases after the second attempt.

\section{3. Secondary outcomes}

After the ILMA placement, ventilation was effective in 1250 cases (85.38\%). After placement of the ILMA, there were minimal leaks in 375 cases $(25.61 \%)$, major leaks in 124 cases (8.46\%), obstruction in 79 cases $(5.39 \%)$ and in 7 cases $(0.47 \%)$ ventilation was impossible. After insertion of the ILMA, the ventilation was effective in 1172 (80.05\%) cases after first attempt, whereas in only $70(4.78 \%)$ cases after the second attempt (Tables 3 and 4).

Regurgitation of gastric contents occurred in 237 cases $(16.18 \%)$, in 187 cases $(12.77 \%)$ before the use of the ILMA, in 25 cases (1.7\%) during the ILMA placement, and in 25 cases (1.7\%) after the ILMA placement. 
Regarding the experience of the FD nurse and the number of training session, we observed that the success rate remained constant over time. The experience of the FD nurses refers to the number of uses of the ILMA in emergency situations before the current intervention. As far as the number of attempts at ILMA is concerned, the OR is less than 1, which means that the more the FD nurse tried to introduce the mask, the less he/she succeeded. On the second attempt, the success rate decreases by 20 times.

After univariate analysis, six variables were introduced in a multivariate analysis. Table 5 summarizes the odds ratio (OR) for each variable used in the statistical analysis. In multivariable analysis, two factors were predictive of a successful tracheal intubation: the performance of the Chandy maneuver $\mathrm{OR}=2.91$ (CI:2.07-3.97) and the number of attempts at intubation $\mathrm{OR}=1.95$ (CI:1.43-2.61). Conversely, the number of attempts at ILMA insertion was predictive of an intubation failure $\mathrm{OR}=0.11$ (CI:0.07-0.17). Effectively, we have 20 times less chances to succeed at intubation on the second attempts at ILMA insertion than the first.

\section{DISCUSSION}

This study demonstrates that, used by a trained FD nurse, the use of the ILMA is feasible and allows effective airway management during OHCA. The characteristics of the study population are similar to the French population suffering of OHCA [23].

The number of intubations considered difficult is smaller in our study [24-29]. The rate of difficult intubations in the paramedic based systems is between $13 \%$ and $26 \%$ [30]. A French work found the same result in a series of 355 intubations in prehospital settings [31].

Previously reported success rate of ETI through the ILMA performed by the novice healthcare providers range from $43 \%$ to $97 \%$ [32-35]. The observed success rate for the insertion of ILMA in this study is similar to that reported previously in studies conducted in the operating room or in the prehospital settings [6, 36-37]. English literature shows quite different results, but most studies have been done on mannequins, cadavers or on patients in the operating room. Keller et al. found that ventilation was satisfactory in $92 \%$ of cases and moderate to satisfactory in $89 \%$ of cases. Major leaks were recorded in 58\% of cases [38]. Very few studies have analyzed the insertion of ILMA in prehospital care [39]. In our study, major leaks were twenty times less present than in the literature. Of note, in these 8 cases the attempt were not performed according to the recommandations.

The ILMA insertion failures are due to the presence of significant bleeding in the upper airway, the corpulence of the patient, anatomical abnormalities (head or neck cancer, hanged, severe head trauma or significant swelling of the tongue). In some situations, inserting the ILMA was possible and ventilation was ample, but intubation was unsuccessful. This is mainly due to the occurence of 
regurgitation, either before or during insertion of the ILMA. Other causes of obstruction founded were insufficient lubrication ( 6 cases), foreign bodies in the mouth (18 cases).

The most common complication was regurgitation which occurred most often after the beginning of resuscitation. Occurence of regurgitation after starting CPR due to the increased intrathoracic pressure during CPR and the fact that patients in prehospital settings are considered full stomach patients. The incidence of regurgitation in this study was lower compared with the results reported in historical data [40].

In 143 cases, a deviation from the protocol was observed. Despite occurence of major leaks after ILMA insertion, the nurses introduced the TT and in 48 cases $(33.56 \%)$ the ventilation after intubation was effective. In this study all 210 cases of failure of ventilation after ILMA insertion were considered as failure of the intubation.

This seems surprising, but only two factors were predictive of a successful intubation: the performance of the Chandy maneuver and the number of attempts at intubation. Conversely, the number of attempts at ILMA insertion was predictive of a failure of intubation. The sitting of Chandy maneuver facilitate the intubation. We can say that the success rate decreases abruptly after the first attempts at ILMA insertion; 20 times on the second attempt.

No factors related to the patient (age, gender, anatomy), intervention (when and where), FD nurses (number of training sessions, experience) significantly influence success of intubation through the ILMA. Usability of ILMA explains this fact. The ILMA is an easy device, with a fast learning curve although of the extra hospital difficult settings. The incurvation radius of ILMA, its stiffness and the ability to apply vertical traction force on the handle of the device probably contribute to the high success rate of placement.

This study has several limitations such as: (1) the goal of the study was limited to the assessment of feasibility and effectiveness of airway control whereas patient survival rate was not evaluated, (2) only clinical criteria were used to judge the success of the ventilation. Capnography, not available at the beginning of this study, was not used as a judgment criteria (3) the success rate of intubation was calculated only for patients in OHCA, (4) this study is an observational study. To account for potential confounding factors multivariate analysis was used.

Future research could compare the impact of ILMA with traditional airway management for OHCA patients in a large randomized controlled trial.

\section{CONCLUSION}


In conclusion, the use of ILMA is feasible and allows effective airway management when performed by trained non medical healthcare professionals during OHCA. In these prospective series of 1464 OHCA, the success rate at ventilation through the ILMA was $85.38 \%$. Among these 1464 cases, $73.63 \%$ could be properly ventilated after tracheal intubation through the ILMA. The most common complication was regurgitation, which was found in $16.18 \%$ of cases, mostly during

BLS. Two factors were predictive of a successful tracheal intubation: the performance of the Chandy maneuver and the number of attempts at intubation. Conversely, the number of attempts at ILMA insertion was predictive of an intubation failure.

\section{Conflict of interest}

None

Number of words: 2994

\section{References}

[1] Soar J, Nolan JP, Böttiger BW, Perkins GD, Lott C, Carli P et al. Adult advanced life support section Collaborators. European Resuscitation Council guidelines for resuscitation 2015: section 3: adult advanced life support. Resuscitation. 2015;95:100-147

[2] Link MS, Berkow LC, Kudenchuk PJ, Halperin HR, Hess EP, Moitra VK, et al. Part7: Adult advanced cardiovascular life support: American Heart Association guidelines update for cardiopulmonary resuscitation and emergency cardiovascular care. Circulation. 2015;132(18)(suppl 2):S444-S464

[3] Jabre P, Penaloza A, Pinero D, Duchateau FX, Borron SW, Javaudin F, et al. Effect on Neurologic Outcomes of Bag-Mask vs Mechanical Ventilation During OHCA. JAMA. 2018;319(8):779-787. doi:10.1001/jama.2018.0156

[4] Benoit JL, Gerecht RB, Steuerwald MT, McMullan JT. Endotracheal intubation versus supraglottic airway placement in out-ofhospital cardiac arrest: a meta-analysis. Resuscitation. 2015; 93: 20-26

[5] Grmec Š. Comparison of three different methods to confirm tracheal tube placement in emergency intubation. Intensive Care Med (2002) 28: 701. https://doi.org/10.1007/s00134-002-1290-x

[6] Lyon R, Ferris J, Young D, McKeown D, Oglesby A, Robertson C. Field intubation of cardiac arrest patients: a dying art? Emerg Med J: EMJ. 2010; 27: 321-323

[7] Jones JH, Murphy MP, Dickson RL, Somerville GG, Brizendine EJ. Emergency physician-verified out-of-hospital intubation: miss rates by paramedics. Acad Emerg Med: Off J Soc Acad Emerg Med. 2004; 11: 707-709

[8] Jemmett ME, Kendal KM, Fourre MW, Burton JH. Unrecognized misplacement of endotracheal tubes in a mixed urban to rural emergency medical services setting. Acad Emerg Med: Off J Soc Acad Emerg Med. 2003; 10: 961-965

[9] Katz SH, Falk JL. Misplaced endotracheal tubes by paramedics in an urban emergency medical services system. Ann Emerg Med. 2001; 37: 32-37

[10] Wang HE, Simeone SJ, Weaver MD, Callaway CW. Interruptions in cardiopulmonary resuscitation from paramedic endotracheal intubation. Ann Emerg Med. 2009; 54 (645-52 e1)

[11] Garza AG, Gratton MC, Coontz D, Noble E, Ma OJ. Effect of paramedic experience on orotracheal intubation success rates. $J$ Emerg Med. 2003; 25: 251-256

[12] Sayre MR, Sakles JC, Mistler AF, Evans JL, Kramer AT, Pancioli AM. Field trial of endotracheal intubation by basic EMTs. Ann Emerg Med. 1998; 31: 228-233

[13] Bradley JS, Billows GL, Olinger ML, Boha SP, Cordell WH, Nelson DR. Prehospital oral endotracheal intubation by rural basic emergency medical technicians. Ann Emerg Med. 1998; 32: 26-32 
[14] Bernhard M, Mohr S, Weigand MA, Martin E, Walther A. Developing the skill of endotracheal intubation: implication for emergency medicine. Acta Anaesthesiol Scand. 2012; 56: 164-171

[15] Youngquist S, Henderson D, Gausche Hill M, Goodrich S, Poore P, Lewis R. Paramedic self efficacy and skill retention in pediatric airway management. Academic Emergency Medicine 2008;15:1295-303

[16] Soar J, Nolan JP, Böttiger BW, Perkins JD, Lott C, Carli P et al. European Resuscitation Council Guidelines for Resuscitation 2015 Section 3. Adult advanced life support. Resuscitation 95 (2015) 100-147

[17] Hubble MW, Wilfong DA, Brown LH, Hertelendy A, Benner RW. A meta-analysis of prehospital airway control techniques. Part II: Alternative airway devices and cricothyrotomy success rates. Prehospital Emergency Care: Official Journal of the National Association of EMS Physicians and the National Association of State EMS Directors 2010;14:515-30

[18] Timmermann A, Braun U, Panzer W, Schlaeger M, Schnitzker M, Graf BM. Out-of-hospital airway management in northern Germany Physician-specific knowledge, procedures and equipment. Der Anaesthesist 2007;56:328-34

[19] Combes X, Aaron E, Jabre P, Leroux B, Lefloch AS, André JY et al. Introduction of the intubating Laryngeal Mask Airway in a prehospital emergency medical unit. Annales Francaises d'Anesthesie et de Reanimation 2006;25:1025-9

[20] Timmermann A, Russo SG, Rosenblatt WH, et al. Intubating laryngeal mask airway for difficult out-of-hospital airway management: a prospective evaluation. British Journal of Anaesthesia 2007;99:286-91

[21] Frascone RJ, Heegaard W, Pippert G, Dries D, Molinari P, Salzman J. Use of the intubating laryngeal mask airway in HEMS. Air Medical Journal 2008;27:182-4

[22] Institut national de la statistique et des études économiques (Accessed 15 April 2018 on https://insee.fr/fr/statistiques/3292643)

[23] Les statistiques publiques- Registre électronique des Arrêts Cardiaques (Accessed 11 march 2018 on https://registreac.org)

[24] Schwartz DE, Matthay MA, Cohen NH. Death and other complications of emergency airway management in critically ill patients. Anesthesiology 1995;82:367-76

[25] Agro F, Brimacombe J, Carassiti M, Marchionni L, Morelli A, Cataldo R. The intubating laryngeal mask: clinical appraisal of ventilation and blind tracheal intubation in 110 patients. Anaesthesia 1998;53:1084-90

[26] Kihara S, Yagushi Y, Brimacombe J, Watanabe S, Taguchi N, Hosoya N. Intubating Laryngeal Mask Airway Size Selection : A randomized triple crossover study in paralysed, anesthetized male and female adult patients. Anesth Analg. 2002;94(4):1023-7

[27] Adnet F, Jouriles NJ, Le Toumelin P, Hennequin B, Taillandier C, Rayeh F, et al. A survey of out-of-hospital emergency intubations in the French Prehospital Medical System: a multicenter study. Ann Emerg Med 1998;32:454-60

[28] Biswas BK, Agarwal B, Bhattacharyya P, Badhani UK, Bhattara B. Intubating laryngeal mask for airway management in lateral decubitus state: comparative study of right and left lateral positions British Journal of Anaesthesia 95(5):715-18

[29] Benger JR, Kirby K, Black S, Brett SJ, Clout M, Lazaroo MJ, et al. Effect of a Strategy of a Supraglottic Airway Device vs Tracheal Intubation During Out-of-Hospital Cardiac Arrest on Functional Outcome: The AIRWAYS-2 Randomized Clinical Trial. JAMA. 2018;320(8):779-791

[30] Chernow B, Holbrook P, D'angona DS, Zaritsky A, Casey LC, Fletcher JR, et al. Epinephrine absorption after intratracheal administration. Anesth Analg 1984;63:829-32

[31] Orliaguet G, Tartière S, Lejay M, Carli P. Prospective in-field evaluation of orotracheal intubation by emergency medical services physicians. JEUR 1997;1:27-32

[32] Timmermann A, Russo SG, Crozier TA, Each C, Mundt B. Novices ventilate and intubate quicker and safer via intubating laryngeal mask than by conventional bag-mask ventilation and laryngoscopy. Anesthesiology 2007;107:570-6

[33] Avidan MS, Harvey A, Chitkara N, Ponte J. The intubating laryngeal mask airway compared with direct laryngoscopy. British Journal of Anaesthesia 1999;83:615-7

[34] Messant I, Lenfant F, Chomel A, Rapenne T, Freysz M. Evaluation of the learning curve of a new intubation techniqueintubating laryngeal mask. Annales Francaises d'Anesthésie et de Réanimation 2002;8:622-6

[35] Burgoyne L, Cyna A. Laryngeal mask vs intubating laryngeal mask: insertion and ventilation by inexperienced resuscitators. Anaesth Intensive Care. 2001(6):604-8

[36] Busch I, Claes D, Thomsin S, Stefanini JL, Fraipont V, Degesves S, et al. Effectiveness of intubating laryngeal mask airway (ILMA Fastrach) used by nurses during out of hospital cardiac arrest resuscitation. Acta Anaesthesiologica Belgica 2009;60:235-8 
[37] Combes X, Jabre P, Margenet A, Merle JC, Leroux B, Dru L, et al. Unanticipated difficult airway management in the prehospital emergency setting. Anesthesiology 2011;114(1):105-10

[38] Keller C, Brimacombe J, Randler C et al. the intubating laryngeal mask airway: effect of handle elevation on efficacy of seal, fiberoptic position, blind intubation and airway protection. Anaest Intensive Care 2000;28:414-9

[39] Tritsch L, Boet S, Pottecher J, Joshi GP, Diemunsch P. Intubating laryngeal mask airway placement by non-physician healthcare providers in management out-of-hospital cardiac arrest : Resuscitation 2014;85: 320-325

[40] Simons RW, Rea TD, Becker LJ, Eisenberg MS. The incidence and significance of emesis associated with out-of-hospital cardiac arrest. Resuscitation 2007;74:427-31 

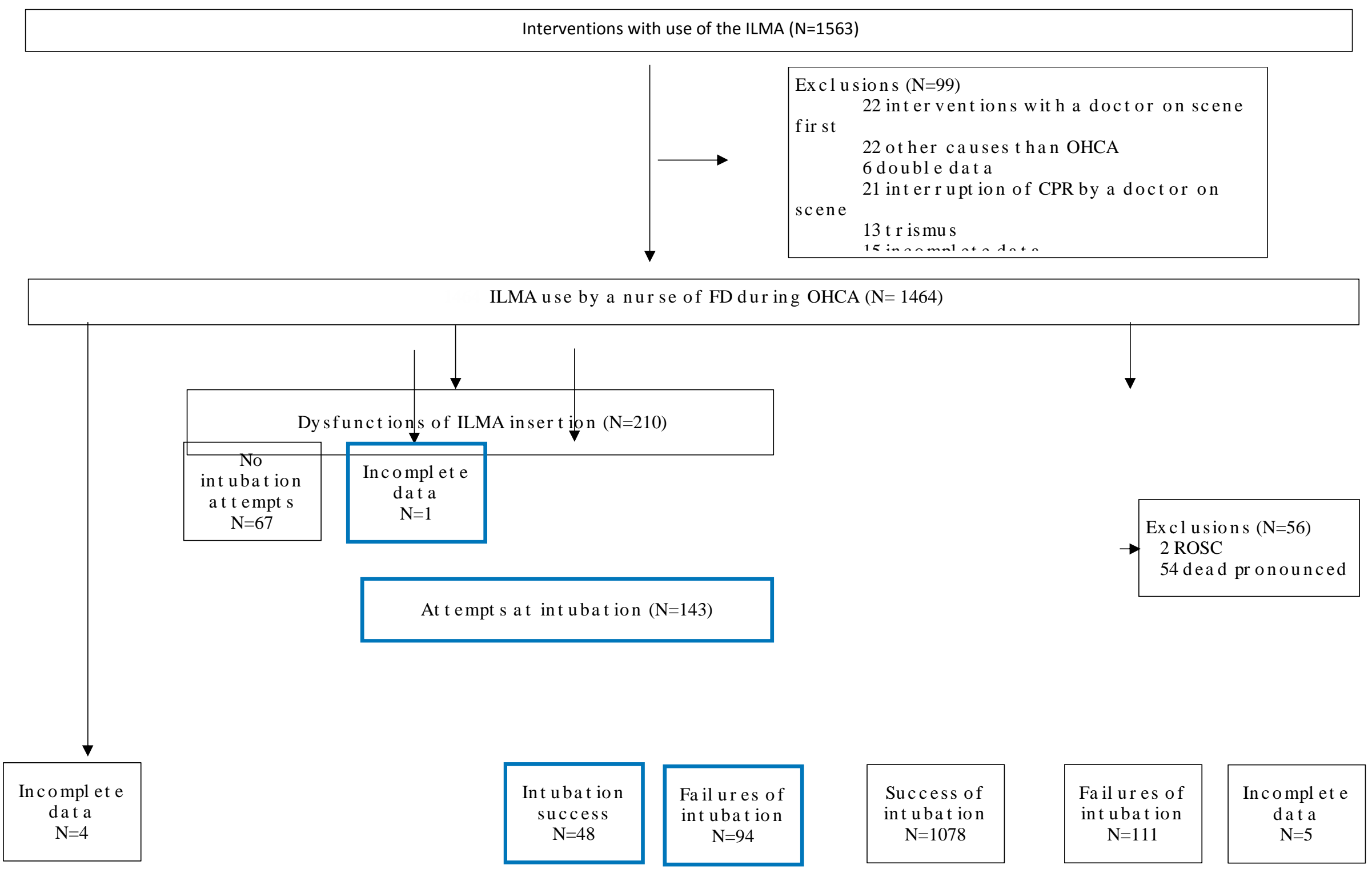

Fig. 1. Fl ow chart (out -of-hospit al cardiac arrest)

ILMA: Int ubat ing Laryngeal Mask Air way; OHCA: Out-of-hospit al cardiac ar rest; CPR: Cardio Pul monary Resuscit at ion; FD: Fir e

Depart ment; ROSC: Ret urn Of Successful Circulation; Deviat ion from the protocol - 
Table 1: Character ist ics of the study populat ion

\section{Patient's gender}

Male

Female

Not recorded

\section{Patient's age}

$<18$ years

$19-49$ years

50-74 y ear s

075 years

Not recorded

Location of the intervention

Pat ient s's home

Publ ic place

Pat ients's workplace

Other

Not recorded

\section{Start time of the intervention}

Day (7 a.m-7 p.m.)

Night (7 p.m-7 a.m.)

Not recorded 


\begin{tabular}{lrr}
\hline & $\mathrm{N}$ & $\%$ \\
\hline Gender of the FD nurses & 162 & 100 \\
Female & 95 & 58.64 \\
Male & 61 & 37.65 \\
Not recorded & 6 & 3.70 \\
Number of interventions according to the gender of the FD nurses & 1464 & 100 \\
Female & 801 & 54.71 \\
Male & 657 & 44.87 \\
Not recorded & 6 & 0.40 \\
Number of interventions according to the number of training sessions & 1464 & 100 \\
1-4 & 712 & 48.63 \\
5-10 & 623 & 42.55 \\
$>10$ & 129 & 8.81 \\
Not recorded & 0 & 0.00
\end{tabular}




\begin{tabular}{lrrrrrrr}
\hline & & Total & & Success & & Failure & Not recorded \\
& $\mathrm{N}$ & $\%$ & $\mathrm{~N}$ & $\%$ & $\mathrm{~N}$ & $\%$ & $\mathrm{~N}$ \\
\hline Total & $\mathbf{1 4 6 4}$ & $\mathbf{1 0 0}$ & $\mathbf{1 2 5 0}$ & $\mathbf{8 5 . 3 8}$ & $\mathbf{2 1 0}$ & $\mathbf{1 4 . 3 4}$ & $\mathbf{4}$ \\
Patient's age & & & & & & & \\
$\quad<18$ years & 11 & 0.75 & 9 & 0.61 & 2 & 0.13 & 0 \\
19-49 years & 189 & 12.90 & 167 & 11.40 & 21 & 1.43 & 1 \\
50-74 years & 637 & 43.51 & 545 & 37.22 & 91 & 6.21 & 1 \\
$\geq 75$ years & 610 & 41.66 & 515 & 35.17 & 93 & 6.35 & 2 \\
$\quad$ Not recorded & 17 & 1.16 & 14 & 0.95 & 3 & 0.20 & 0
\end{tabular}

Number of interventions

according to the gender of the FD nurses

Female FD nurses
Male FD nurses
Not recorded

Number of uses of the ILMA in emergency situations

$$
<5
$$$$
\text { 5-10 }
$$$$
>10
$$

Not recorded

Number of interventions according to the number of training sessions

$$
1
$$$$
2
$$$$
3
$$$$
4
$$

$\geq 5$

Not recorded

The location of the intervention

Patient's home
Public place
Patient's workplace
Other
Not recorded

\section{Start time of the intervention}

$$
\begin{aligned}
& \text { Day (7 a.m-7 p.m) } \\
& \text { Night (7 p.m-7 a.m) } \\
& \text { Not recorded }
\end{aligned}
$$

Number of attempts to introduce the ILMA

$$
\begin{aligned}
& 1 \\
& 2 \\
& 3 \\
& 4
\end{aligned}
$$$$
5
$$

Not recorded

Difficult intubation criteria

$$
\text { Yes }
$$$$
\text { No }
$$

Not recorded

FD: Fire Department; ILMA: Intubating Laryngeal Mask Airway

$\begin{array}{rrrrrrr}801 & 54.71 & 679 & 46.37 & 118 & 8.06 & 4 \\ 657 & 44.87 & 566 & 38.66 & 91 & 6.21 & 0 \\ 6 & 0.40 & 5 & 0.34 & 1 & 0.06 & 0\end{array}$

$\begin{array}{rrrrrrr}458 & 31.28 & 377 & 25.75 & 78 & 5.32 & 3 \\ 339 & 23.15 & 283 & 19.33 & 56 & 3.82 & 0 \\ 666 & 45.49 & 589 & 40.23 & 76 & 5.19 & 1 \\ 1 & 0.06 & 1 & 0.06 & 0 & 0.00 & 0\end{array}$

$\begin{array}{rrrrrrr}182 & 12.43 & 145 & 9.90 & 37 & 2.52 & 0 \\ 202 & 13.79 & 162 & 11.06 & 40 & 2.73 & 0 \\ 186 & 12.70 & 156 & 10.65 & 30 & 2.04 & 0 \\ 142 & 9.69 & 120 & 8.19 & 19 & 1.29 & 3 \\ 752 & 51.36 & 667 & 45.56 & 84 & 5.73 & 1 \\ 0 & 0.00 & 0 & 0.00 & 0 & 0.00 & 0\end{array}$

$\begin{array}{rrrrrrr}1138 & 77.73 & 965 & 65.91 & 172 & 11.74 & 1 \\ 181 & 12.36 & 155 & 10.58 & 24 & 1.63 & 2 \\ 9 & 0.61 & 8 & 0.54 & 0 & 0.00 & 1 \\ 129 & 8.81 & 116 & 7.92 & 13 & 0.88 & 0 \\ 7 & 0.47 & 6 & 0.40 & 1 & 0.06 & 0\end{array}$

$\begin{array}{lllllll}952 & 65.02 & 810 & 55.32 & 140 & 9.56 & 2\end{array}$

$\begin{array}{lllllll}492 & 33.60 & 427 & 29.16 & 63 & 4.30 & 2\end{array}$

$\begin{array}{lllllll}20 & 1.36 & 13 & 0.88 & 7 & 0.47 & 0\end{array}$

$\begin{array}{rrrrrrr}1285 & 87.77 & 1172 & 80.05 & 112 & 7.65 & 1 \\ 158 & 10.79 & 70 & 4.78 & 86 & 5.87 & 2 \\ 14 & 0.95 & 5 & 0.34 & 8 & 0.54 & 1 \\ 2 & 0.13 & 1 & 0.06 & 1 & 0.06 & 0 \\ 1 & 0.06 & 0 & 0.00 & 1 & 0.06 & 0 \\ 4 & 0.27 & 2 & 0.13 & 2 & 0.13 & 0\end{array}$

$\begin{array}{rrrrrrr}35 & 2.39 & 21 & 1.43 & 14 & 0.95 & 0 \\ 1425 & 97.33 & 1225 & 83.67 & 196 & 13.38 & 4 \\ 4 & 0.27 & 4 & 0.27 & 0 & 0.00 & 0\end{array}$


ILMA:Int ubat ing Lar yngeal Mask Airway 
* 210 fail ures because the vent il ation $t h r o u g h t h e$ ILMA was ineffective and 111 because the vent il at ion $t h r o u g h t h e t r a c h e a l ~ t u b e$ 
Success

Failure*

No CPR**

Not recorded

\begin{tabular}{rrrrrrrrr} 
& $\mathrm{N}$ & $\%$ & $\mathrm{~N}$ & $\%$ & $\mathrm{~N}$ & $\%$ & $\mathrm{~N}$ & $\mathrm{~N}$ \\
\hline Total & 1464 & 100 & 1078 & 73.63 & 321 & 21.92 & 56 & 9
\end{tabular}

\section{Patient's age}

$<18$ years

19-49 years

50-74 years

$\geq 75$ years

Not recorded

Gender of the FD nurses

Female

Male

Not recorded

Number of uses of the

ILMA in emergency situations

$$
<5
$$

5-10

$>10$

Not recorded

Number of training sessions

1

$$
2
$$

3

4

$\geq 5$

Not recorded

The location of the

intervention

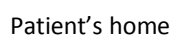

Public place, workplace

Other

Not recorded

Start time of the

intervention

Day

Not recorded

Difficult intubation criteria

No

Yes

Not recorded

Number of attempts to introduce the ILMA

1

$$
2
$$$$
3
$$$$
4
$$$$
\geq 5
$$

Not recorded

The performance of the Chandy maneuver

Yes
No recorded

Number of attempts at intubation

was ineffective

$\begin{array}{rrrrrrrr}11 & 0.75 & 7 & 0.47 & 2 & 0.13 & 1 & 1 \\ 189 & 12.90 & 143 & 9.76 & 34 & 2.32 & 9 & 3 \\ 637 & 43.51 & 461 & 31.48 & 152 & 10.38 & 23 & 1 \\ 610 & 41.66 & 457 & 31.21 & 129 & 8.81 & 20 & 4 \\ 17 & 1.16 & 10 & 0.68 & 4 & 0.27 & 3 & 0 \\ 801 & 54.71 & 567 & 38.72 & 186 & 12.70 & & 2 \\ 657 & 44.87 & 509 & 34.76 & 132 & 9.01 & 16 & 7 \\ 6 & 0.40 & 2 & 0.13 & 3 & 0.20 & 1 & 0\end{array}$

$\begin{array}{rrrrrrrr}458 & 31.28 & 307 & 20.96 & 121 & 8.26 & 25 & 5 \\ 339 & 23.15 & 244 & 16.66 & 82 & 5.60 & 12 & 1 \\ 666 & 45.49 & 527 & 35.99 & 118 & 8.06 & 18 & 3 \\ 1 & 0.06 & 0 & 0.00 & 0 & 0.00 & 1 & 0\end{array}$

$\begin{array}{rrrrrrrl}182 & 12.43 & 119 & 8.12 & 54 & 3.68 & 9 & 0 \\ 202 & 13.79 & 142 & 9.69 & 54 & 3.68 & 6 & 0 \\ 186 & 12.70 & 132 & 9.01 & 44 & 3.00 & 9 & 1 \\ 142 & 9.69 & 106 & 7.24 & 29 & 1.98 & 3 & 4 \\ 752 & 51.36 & 579 & 39.54 & 140 & 9.56 & 29 & 4 \\ 0 & 0.00 & 0 & 0.00 & 0 & 0.00 & 0 & 0\end{array}$

$\begin{array}{rrrrrrrr}1138 & 77.73 & 828 & 56.55 & 261 & 17.82 & 45 & 4 \\ 190 & 12.97 & 140 & 9.56 & 37 & 2.52 & 8 & 5 \\ 129 & 8.81 & 104 & 7.10 & 22 & 1.50 & 3 & 0 \\ 7 & 0.47 & 6 & 0.40 & 1 & 0.06 & 0 & 0\end{array}$

$\begin{array}{rrrrrrrr}952 & 65.02 & 695 & 47.47 & 222 & 15.16 & 30 & 5 \\ 492 & 33.60 & 372 & 25.40 & 90 & 6.14 & 26 & 4 \\ 20 & 1.36 & 11 & 0.75 & 9 & 0.61 & 0 & 0\end{array}$

$\begin{array}{rrrrrrrr}1425 & 97.33 & 1060 & 72.40 & 301 & 20.56 & 55 & 9 \\ 35 & 2.39 & 15 & 1.02 & 19 & 1.29 & 1 & 0 \\ 4 & 0.27 & 3 & 0.20 & 1 & 0.06 & 0 & 0\end{array}$

$\begin{array}{rrrrrrrr}1285 & 87.77 & 1025 & 70.01 & 204 & 13.93 & 51 & 5 \\ 158 & 10.79 & 49 & 3.34 & 102 & 6.96 & 4 & 3 \\ 14 & 0.95 & 4 & 0.27 & 9 & 0.61 & 0 & 1 \\ 2 & 0.13 & 0 & 0 & 2 & 0.13 & 0 & 0 \\ 1 & 0.06 & 0 & 0 & 1 & 0.06 & 0 & 0 \\ 4 & 0.27 & 0 & 0 & 3 & 0.20 & 1 & 0\end{array}$

\begin{tabular}{|c|c|c|c|c|c|c|c|}
\hline 109 & 7.44 & 0 & 0.00 & 52 & 3.55 & 56 & 1 \\
\hline 1186 & 81.01 & 1020 & 69.67 & 163 & 11.13 & 0 & 3 \\
\hline 124 & 8.46 & 50 & 3.41 & 72 & 4.91 & 0 & 2 \\
\hline 19 & 1.29 & 5 & 0.34 & 14 & 0.95 & 0 & 0 \\
\hline 0 & 0.00 & 0 & 0.00 & 0 & 0.00 & 0 & 0 \\
\hline 1 & 0.06 & 0 & 0.00 & 1 & 0.06 & 0 & 0 \\
\hline 25 & 1.70 & 3 & 0.20 & 19 & 1.29 & 0 & ? \\
\hline
\end{tabular}

$\begin{array}{rrrrrrrr}276 & 18.85 & 186 & 12.70 & 87 & 5.94 & 2 & 1 \\ 1090 & 74.45 & 882 & 60.24 & 184 & 12.5 & 22 & 2 \\ 98 & 6.69 & 10 & 0.68 & 50 & 3.41 & 32 & 6\end{array}$


** After the insertion of the ILMA, but before the insertion of the $\mathrm{tracheal} t \mathrm{ube}$, a doct or was on scene and he stop the cardio pul monary resuscit at ion (CPR) 
Table 5: Un ivar iate and mult ivar iate analysis

\begin{tabular}{|c|c|c|c|c|c|c|c|}
\hline & \multicolumn{3}{|c|}{ Univariat e } & \multicolumn{3}{|c|}{ Mul tivariate } & \multirow{2}{*}{$\begin{array}{c}\text { Target } \\
\text { range }\end{array}$} \\
\hline & OR & CI $(95 \%)$ & $\begin{array}{r}\operatorname{Pr}(\mathrm{OR} \\
)\end{array}$ & OR & CI (95\%) & $\begin{array}{r}\operatorname{Pr}(\mathrm{OR} \\
)\end{array}$ & \\
\hline \multicolumn{8}{|l|}{ Pat ient s's age } \\
\hline$<18$ years & 1.29 & $0.22-5.06$ & 0.09 & & & & $0.9-1.1$ \\
\hline $19-45$ y ea r s & 1.00 & $1.00-1.00$ & 1.00 & & & & $0.0-0.0$ \\
\hline $50-74$ years & 0.86 & $0.56-1.23$ & 0.26 & & & & $0.9-1.1$ \\
\hline Q75 y ea r s & 0.99 & $0.65-1.43$ & 0.38 & & & & $0.9-1.1$ \\
\hline Gender of the FDnurses & 0.81 & $0.58-1.10$ & 0.18 & & & & $0.9-1.1$ \\
\hline $\begin{array}{l}\text { Start time of the intervention: } \\
\text { day/night }\end{array}$ & 0.93 & $0.70-1.22$ & 1.00 & & & & $0.7-0.9$ \\
\hline \multicolumn{8}{|l|}{ Intervention's pl ace } \\
\hline Pat ient's home & 1.00 & $1.00-1.00$ & 1.00 & & & & $0.0-0.0$ \\
\hline Public place & 0.78 & $0.46-1.26$ & 0.12 & & & & $0.5-1$ \\
\hline Pat ient's workplace & 3.70 & $0.50-16.31$ & 0.08 & & & & $0.5-1$ \\
\hline Duration of the intervention & 0.99 & $0.97-1.01$ & 1.00 & 0.99 & $0.97-1.01$ & 1.00 & $0.9-1.1$ \\
\hline Number of training sessions & 1.02 & $0.98-1.06$ & 0.87 & 1.01 & $0.96-1.06$ & 0.67 & $1-2$ \\
\hline Number of sit $t$ ing in emergency & 1.00 & $0.99-1.00$ & 0.67 & 0.99 & $0.98-1.00$ & 0.31 & $1-2$ \\
\hline The performance of Chandy manoeuver & 2.23 & $1.56-3.10$ & 0.01 & 2.91 & 2.07-3.97 & 0.01 & $1-2$ \\
\hline Number of attempts at ILMA & 0.13 & $0.09-0.18$ & 0.00 & 0.11 & $0.07-0.17$ & 0.00 & $1-1.5$ \\
\hline Number of attempts at intubation & 1.25 & $0.95-1.64$ & 0.92 & 1.95 & 1.43-2.61 & 0.59 & $1-2$ \\
\hline
\end{tabular}

